\title{
Représenter le chantier
}

Exposition L'Art du chantier. Construire et démolir $\mathrm{du} \mathrm{XVI}^{\mathrm{e}}$ au XXI ${ }^{\mathrm{e}}$ siècle, Cité de l'architecture et du patrimoine, Paris, 9 novembre 2018 - 11 mars 2019

\section{Valérie Nègre}

\section{OpenEdition Journals}

\section{Édition électronique}

URL : http://journals.openedition.org/artefact/4226

DOI : 10.4000/artefact.4226

ISSN : 2606-9245

\section{Éditeur :}

Association Artefact. Techniques histoire et sciences humaines, Presses universitaires du Midi

\section{Édition imprimée}

Date de publication : 15 octobre 2019

Pagination : 197-199

ISBN : 978-2-8107-0650-1

ISSN : $2273-0753$

\section{Référence électronique}

Valérie Nègre, «Représenter le chantier », Artefact [En ligne], 10 | 2019, mis en ligne le 06 août 2020, consulté le 27 novembre 2020. URL : http://journals.openedition.org/artefact/4226 ; DOI : https:// doi.org/10.4000/artefact.4226

\section{(ब) $\odot \Theta$}

Artefact, Techniques, histoire et sciences humaines est mise à disposition selon les termes de la Licence Creative Commons Attribution - Pas d'Utilisation Commerciale - Pas de Modification 4.0 International. 


\section{Représenter le chantier}

\section{Exposition L'Art du chantier. Construire et démolir du $X_{X I}^{e}$ au XXI siècle, Cité de l'architecture et du patrimoine, Paris, 9 novembre 2018 - 11 mars 2019}

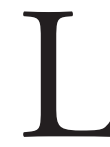

'exposition L'Art du chantier présentée à la Cité de l'architecture et du patrimoine porte sur la manière dont les hommes ont, en Occident, depuis la Renaissance, regardé, conçu et imaginé le lieu où l'on bâtit. La grande diversité des observateurs, la multiplicité des images et la variété des publics auxquels elles s'adressent montrent qu'il s'agit d'un thème fort, dépassant largement les mondes de l'architecture et de la technique. Au point que l'on peut se demander si la représentation du chantier ne serait pas plus captivante que celle de l'œuvre bâtie, plus vivante, plus puissante que celle de l'édifice achevé.

D'un côté, le chantier est perçu comme une source de nuisances et de dangers, un espace encombré et encombrant, dont atteste l'expression " Quel chantier ! ». De l'autre, il exerce une fascination continue depuis des temps anciens. Ses éléments mobiles et ses dispositifs temporaires le rapprochent d'un spectacle de rue ; mais surtout il est, par son caractère provisoire et non fini, une métaphore même du possible et du virtuel.

L'exposition confronte différents regards. Elle réunit un ensemble d'œuvres et de documents produits par des artistes, des illustrateurs, des amateurs, mais aussi par ceux qui travaillent sur les lieux : ingénieurs (Fig. VII, cahier couleur), architectes, entrepreneurs et - ce qui est plus rare - ouvriers, à travers des ex-voto (Fig. VIII, cahier couleur) ou des chefs-d'œuvre réalisés par les Compagnons charpentiers des Devoirs du Tour de France.

99 Valérie Nègre « Représenter le chantier », Artefact, 10, 2019, p. 197-200. 
Elle s'achève avec les témoignages de trois constructeurs contemporains : Patrick Bouchain, Marc Mimram et Martin Rauch qui placent le processus constructif au cœur de leur travail.

Le chantier comme fait culturel et social, et, si l'on peut dire, comme volonté collective et comme représentation, n’a jamais été étudié dans sa globalité à travers des sources figurées variées, même s'il fait aujourd'hui l'objet d'un fort regain d'intérêt. Pris sous l'angle de la représentation visuelle, le thème a jusqu'à présent essentiellement intéressé les domaines de la photographie et de l'art contemporain. Il faut dire que le chantier est un grand sujet de la photographie depuis ses origines. Les principales entreprises constructives du XIX ${ }^{\mathrm{e}}$ siècle ont été soigneusement documentées. Jusqu'à présent, l'attention des spécialistes s'est plutôt focalisée sur les ouvrages d'art et les monuments publics ainsi que sur les auteurs et les institutions à l'origine de ces campagnes photographiques. De leur côté, les spécialistes de l'art contemporain ont examiné la façon dont, depuis le $\mathrm{XIX}^{\mathrm{e}}$ siècle, les artistes, les chorégraphes, les cinéastes ont puisé une énergie et des formes dans le sujet.

Face à un corpus si hétéroclite, il semblait important d'inviter, et des spécialistes de l'art, et des spécialistes des techniques à participer à cette entreprise collective (voir le catalogue de l'exposition). Les historiens et les praticiens de la construction prêtent depuis longtemps attention aux pratiques. Ils savent reconnaître et comprendre les objets et les dispositifs techniques et repérer les décalages entre la réalité et la fiction. De leur côté, les historiens de l'art sont familiers des corpus visuels, même s'ils n'ont pas à ce jour accordé une importance et une valeur significative au chantier, comme ils l'ont fait par ailleurs pour l'atelier du peintre et celui du sculpteur. Pourtant, comme le suggèrent bien des sources anciennes, les deux espaces sont proches. Un grand dessin inédit exposé, titré : "Vue perspective du chantier " exécuté par Jules Doré à la demande la Société des ouvriers charpentier de La Villette en 1889, indique clairement que le mot désignait encore au début du $\mathrm{XIX}^{\mathrm{e}}$ siècle un atelier à ciel ouvert. L'aquarelle ne donne pas à voir un édifice en construction, mais bien l'endroit où les entrepreneurs entreposaient et travaillaient les matériaux avant d'être mis en œuvre dans les bâtiments (Fig. IX, cahier couleur).

L'exposition propose une lecture multiple du sujet : technique, mais également sociale, politique et artistique. Le parcours met en avant les thèmes 
les plus courants qui traversent l'ensemble de la période ainsi qu'un petit nombre d'artistes qui ont accordé au sujet une attention particulière. Elle révèle aussi en filigrane ce qui n'est pas montré : le travail des femmes et des enfants par exemple. Alors que les sources d'archives attestent leur présence ancienne, les femmes n'apparaissent dans les images de chantier que tardivement, au moment de la Première Guerre mondiale semble-t-il. Quant aux enfants manœuvres, ils ne sont presque jamais figurés.

La première partie rassemble des vues qui se focalisent sur les opérations techniques (Grands travaux ; Espaces, procédés et machines ; Mécanisation et industrialisation ; Des hommes et des gestes). La deuxième met en lumière les enjeux politiques et sociaux du lieu où l'on bâtit (Démolitions ; Le Chantier dans la ville ; Visites et visiteurs ; Luttes sociales). Enfin la troisième traite des rapports entre art et chantier. Elle montre comment celui-ci contribue, par son caractère transitoire et évolutif, à transformer l'art et l'architecture (Tester et expérimenter ; Esthétiques du chantier). Par ce rassemblement sans précédent de documents et d'œuvres visuelles (433 numéros), c'est à une réflexion sur la dimension anthropologique de l'acte d'édifier et sur l'importance de cet acte pour nos sociétés que cette exposition entend contribuer. 
Exposition L'Art du chantier. Construire et démolir du XVI au XXI siècle, Cité de l'architecture et du patrimoine, Paris, 9 novembre 2018 - 11 mars 2019

Commissariat principal : Valérie Nègre et Marie Hélène Contal ; Assistées de Diane Aymard, doctorante ;

L'exposition est accompagnée d'un catalogue qui reprend les grands thèmes traités dans le parcours. Il comprend en outre, en fin de volume, la liste exhaustive des 433 ouvres exposées.

Nègre Valérie (dir.), L'Art du chantier. Construire et démolir du $X V I^{e}$ au XXI siècle, Snoeck, Paris, 2018, 285 p.

Avec la participation de Laurent Baridon (Université Lumière Lyon 2), Robert Carvais (CNRS - Université Paris Nanterre), Marie-Hélène Contal (Cité de l'architecture et du patrimoine, Paris), Olivier Delarozière (Musée des arts et métiers, Paris), Christophe Feuillerat (Bibliothèque nationale de France), Marie Gaimard (École nationale supérieure d'architecture, La Villette), Stephan Hölzer (ETH, Zürich), Hélène Jannière (Université Rennes 2), Guy Lambert (École nationale supérieure d'architecture, Paris Belleville), Charlotte Leblanc (DRAC Bourgogne), Bertrand Lemoine (CNRS), Anne de Mondenard (Musée Carnavalet, Paris), Nicola Navone (Archivio del Moderno), Jean-Luc de Ochandiano (Laboratoire de recherche historique Rhône-Alpes), Émilie d'Orgeix (École pratique des hautes études), Valérie Nègre (Université Paris 1 Panthéon-Sorbonne), Antoine Picon (Harvard University/École nationale des ponts et chaussées), Christoph Rauhut (Berlin State Conservation Authority), Bruno Reichlin (Université de Genève), Frances Sands (Sir John Soane Museum, Londres), Isabelle Warmoes (Musée des Plans-reliefs, Paris) et Ariane Wilson (École nationale supérieure d'architecture, Paris Malaquais). 